\title{
A procedure for preserving and vouchering butterflies and moths for large-scale museum-based molecular research
}

Soowon Cho, Samantha W Epstein, Kim Mitter, Chris A Hamilton, David Plotkin, Charles Mitter, Akito Y Kawahara

Butterflies and moths (Lepidoptera) comprise significant portions of the world's natural history collections, but a standard for the preservation of their tissues for molecular research is largely lacking. Lepidoptera have traditionally been spread on mounting boards to display wing patterns and colors, which are often important for species identification. Many molecular phylogenetic studies have used legs from pinned specimens as the primary source for DNA in order to preserve a morphological voucher, but the amount of available tissue is often limited. Preserving an entire specimen in a cryogenic freezer is ideal for DNA preservation, but without an easily accessible voucher it can make specimen identification, verification, and morphological work difficult. Here we present a procedure that creates accessible and easily visualized "wing vouchers" of individual Lepidoptera specimens, and preserves the remainder of the insect in a cryogenic freezer for molecular research. Wings are preserved in protective holders so that both dorsal and ventral patterns and colors can be easily viewed without further damage. Our wing vouchering system has been implemented at the University of Maryland (AToL Lep Collection) and the University of Florida (Florida Museum of Natural History, McGuire Center of Lepidoptera and Biodiversity), which house two of the largest Lepidoptera morphological and molecular collections in the world. 
1 Title: A procedure for preserving and vouchering butterflies and moths for large-scale museum-

2 based molecular research

3

4 Authors:

5 Soowon Cho ${ }^{1,2 *}$, Samantha W. Epstein ${ }^{3}, \mathrm{Kim} \mathrm{Mitter}^{2}$, Chris A. Hamilton ${ }^{3}$, David Plotkin ${ }^{3}$,

6 Charles Mitter $^{2}$, Akito Y. Kawahara ${ }^{3 *}$

7

8 Institutional Affiliations:

$9 \quad{ }^{1}$ Department of Plant Medicine, Chungbuk National University, Cheongju, Korea

10

11 2Department of Entomology, University of Maryland, College Park, MD 20742, United States of 12 America

13

$14{ }^{3}$ Florida Museum of Natural History, University of Florida, Gainesville, FL 32611 United States 15 of America

16

17 *shared corresponding authors

18 


\section{Abstract}

21 Butterflies and moths (Lepidoptera) comprise significant portions of the world's natural history

22 collections, but a standard for the preservation of their tissues for molecular research is largely

23 lacking. Lepidoptera have traditionally been spread on mounting boards to display wing patterns

24 and colors, which are often important for species identification. Many molecular phylogenetic

25 studies have used legs from pinned specimens as the primary source for DNA in order to

26 preserve a morphological voucher, but the amount of available tissue is often limited. Preserving

27 an entire specimen in a cryogenic freezer is ideal for DNA preservation, but without an easily

28 accessible voucher it can make specimen identification, verification, and morphological work

29 difficult. Here we present a procedure that creates accessible and easily visualized "wing

30 vouchers" of individual Lepidoptera specimens, and preserves the remainder of the insect in a

31 cryogenic freezer for molecular research. Wings are preserved in protective holders so that both

32 dorsal and ventral patterns and colors can be easily viewed without further damage. Our wing

33 vouchering system has been implemented at the University of Maryland (AToL Lep Collection)

34 and the University of Florida (Florida Museum of Natural History, McGuire Center of

35 Lepidoptera and Biodiversity), which house two of the largest Lepidoptera morphological and

36 molecular collections in the world. 


\section{Introduction}

With over 157,000 described species, Lepidoptera (butterflies and moths) is one of the most diverse insect orders (van Nieukerken et al. 2011). Lepidoptera are frequently collected for their beauty; consequently, they comprise significant portions of the world's natural history collections. Historically, butterflies and moths have been preserved as dried, pinned specimens with their wings spread. This allows for aesthetically pleasing displays and access to genitalia, the dissection of which has been a standard for Lepidoptera taxonomy for centuries (Knolke et al. 2005).

6 Within the last several decades, there has been a growing need for researchers to obtain molecular data from butterfly and moth specimens. Many lepidopterists remove legs from specimens and extract DNA from them (e.g., Hebert et al. 2004; Knolke et al. 2005; Zimmermann et al. 2000), so that tissues can be obtained without compromising diagnostic morphological structures. However, dried and pinned specimens are often low in DNA yield, and many pinned Lepidoptera specimens have been rehydrated, either with direct injection of water or with a relaxing chamber, which can result in degraded DNA. In order to obtain sufficient quantities of DNA, destructive sampling approaches have been proposed for museum specimens, but such approaches can often result in little or no morphological voucher (Wandeler et al. 2007).

6 At a time when large amounts of purified DNA are needed for the increasing number of molecular studies, museums are uniquely positioned to serve as an important resource for the preservation of high-quality molecular-grade tissues. A challenge to many molecular tissue preservation methods is that these approaches damage key structures needed for identification of the organism. Brower $(1994 ; 1996 ; 2000)$ described a procedure for Lepidoptera tissue 
61 preservation in which the head and thorax are preserved in $\geq 95 \%$ ethanol and stored in a deep

62 freezer. He suggested that the wings, abdomen, antennae, and palpi are glued to a $1 \times 3 \mathrm{~cm}$ card,

63 and designated as morphological vouchers to enable examination of diagnostic characters. While

64 Brower's approach can yield potentially large amounts of nucleic acids from the head and

65 thorax, appendages glued to a card can only be viewed from a single perspective (i.e., only one

66 wing surface can be examined) and the amount of sample preparation time can be long, making

67 it difficult to implement for large collections that wish to create tissue collections rapidly.

68 Here we present an effective procedure for storing Lepidoptera tissues that has been

69 successfully implemented at the University of Maryland (UMD) AToL Lep Collection as part of

70 the Lepidoptera Tree of Life (LepTree) project (e.g., Bazinet et al. 2013; Cho et al. 2011; Regier

71 et al. 2009; 2013; 2015), and at the University of Florida's McGuire Center for Lepidoptera and

72 Biodiversity (MGCL), Florida Museum of Natural History (FLMNH), one of the largest

73 Lepidoptera collections in the United States (Kawahara et al. 2012). Wings are accessioned and

74 saved in clear plastic coin holders or laminated cards, and the remainder of the specimen is

75 preserved as a tissue sample in a cryogenic freezer and stored for future use. This approach

76 enables easy examination and digitization using a flatbed scanner of both dorsal and ventral wing

77 surfaces, preservation of diagnostic body parts such as genitalia, and long-term storage of high-

78 quality tissues for nearly all body regions for molecular analysis.

79

80 Methods

81 1. Specimen sampling. Lepidoptera are collected in many different ways. Butterflies are typically

82 caught with aerial nets, and nocturnal moths are often lured to a single site using a visual or

83 olfactory stimulus, such as ultraviolet light or fermenting sugar, and trapped in a jar containing a 
84 killing agent (e.g., Brehm \& Axmacher 2006; Utrio 1983). Although common Lepidoptera

85 killing agents (i.e., cyanide, ethyl acetate) have been shown not to impact recovery of mtDNA

86 COI barcodes (Willows-Munro \& Schoeman 2015), there is still some debate as to whether they

87 can have deleterious effects on genomic DNA (Dean \& Ballard 2001; Dillon et al. 1996). If RNA

88 is required (e.g., for transcriptomics), the best practice is to collect the specimen without using a

89 killing agent and flash-freeze it in an ultra-cold freezer or grind it in a buffer such as Thermo-

90 Fisher Scientific's RNA-Later ${ }^{\circledR}$, as RNA will degrade rapidly following death. (We note,

91 however, that material preserved in $95-100 \%$ ethanol and stored at $-80^{\circ} \mathrm{C}$ has also yielded

92 satisfactory results for RNA-Seq; Bazinet et al. 2013).

93 If the goal is to obtain gDNA, individual specimens can be preserved in a more cost-

94 effective manner by placing them into tubes containing $\geq 95 \%$ ethanol and storing them in a -

$9580^{\circ} \mathrm{C}$ freezer. At UMD and MGCL, specimens are stored individually in $2 \mathrm{~mL}$ microcentrifuge

96 tubes, $15 \mathrm{~mL}$ centrifuge tubes, or $50 \mathrm{~mL}$ centrifuge tubes, depending on the specimen's

97 wingspan and body size. Multiple specimens from the same location can be stored together in a

98 single Nasco ${ }^{\circledR}$ Whirl-Pak ${ }^{\mathrm{TM}}$ bag containing $\geq 95 \%$ ethanol and a locality label (Fig. 1A). For

99 each Whirl-Pak, the number of specimens is usually kept to $\leq 10$, in order to ensure that the

100 ethanol does not become too diluted. We typically use the $18 \mathrm{oz}$. (532 ml) capacity 4-1/2" W x

101 9" L (11.5 cm x $23 \mathrm{~cm})$ Whirl-Pak bag, but other sizes can be used.

102

The use of a Whirl-Pak for mass storage of specimens is not unique to this research. The

103 technique is often applied to large-scale insect diversity studies. If specimens are kept in Whirl-

104 Paks in dense numbers, the approach does not significantly harm specimens, as the number of

105 specimens can prevent movement of samples inside the bag. We change the ethanol in the bag

106 while in the field to prevent ethanol dilution, and after several thousand PCRs, we have not 
107 experienced any cross-specimen DNA contamination with this procedure. Unfortunately, some

108 scales can be lost in the ethanol, with additional ones during the drying process and the

109 placement of the specimens into the protective sleeves (note the detached red scales in the clear

110 window, Fig. 1J). Although the procedure cannot prevent some damage, we find that our

111 approach provides the best compromise between large-scale biodiversity sampling, a

112 morphological voucher, and tissues usable for next-gen DNA sequencing. For best DNA quality

113 and quantity results, specimens stored in ethanol should be deposited in a freezer with a

114 temperature below $-80^{\circ} \mathrm{C}$ as soon as possible.

115 Alternatively, Lepidoptera specimens can be collected, papered in a glassine envelope,

116 and dried. While there are numerous methods for drying Lepidoptera (e.g., placing specimens in

117 a drying chamber), a simple method is to place the glassine envelope containing the Lepidoptera

118 specimen within a small, airtight, Ziploc $®$ sandwich bag containing silica gel beads, which act as

119 a desiccant. For best DNA recovery, specimens should be collected fresh and placed in the

120 envelope before or very shortly after death, as humidity after specimen death can lead to nucleic

121 acid degradation. At MGCL, we use a 5:1 mixture of clear and colored silica gel beads; the latter

122 change color when saturated with water, thus serving as an indicator that helps determine when

123 the gel needs to be replaced. Two tablespoons of the silica gel mixture is added to each Ziploc

124 bag, placing no more than 20 lepidopterans in a single bag. Silica gel is a great resource because 125 it can be heated and dried for multiple uses.

126

127 2. Labeling. Each specimen is given a unique alphanumeric accession code (e.g., "LEP-12345",

128 Fig. 1E) that associates its tissue sample with its corresponding wing voucher. The code is also

129 entered into a collections database and printed on acid-free cardstock with 11-point bold Arial 
130 font. Using the same acid-free cardstock, a locality label is printed in 5-point Arial font. Copies

131 of the code and locality labels are inserted into the specimen tube, and a duplicate copy of each

132 label is saved so that it can be attached to the corresponding wing voucher (see Step 5, below). If

133 specimens were initially collected in a Whirl-Pak, they are gently rinsed with $\geq 95 \%$ ethanol

134 after removal from the Whirl-Pak bag and subsequently transferred to individual tubes

135 containing $\geq 95 \%$ ethanol (see Step 3, below). Ethanol is stored in a $-20^{\circ} \mathrm{C}$ freezer prior to

136 application in order to prevent warming when the tissues are transferred.

137

138

139 3. Wing removal. This step requires a sterile surface, such as a sterile plastic petri dish. The dish,

140 sterilized dissecting scissors and surgical forceps should be prepared before specimens are

141 removed from the freezer, in order to minimize the specimens' exposure to room temperature.

142 When ready for wing removal, a specimen is transferred from its container and placed on the

143 sterile surface. Scissors are used to cut the right forewing and hindwing at the wing base, and

144 both wings are gently removed with forceps (Fig. 1B, C). The left pair of wings is not removed;

145 it serves as a secondary identification measure, should the first pair become misplaced. For

146 consistency, we use the right wings as vouchers, unless severe damage to the right wings renders

147 them useless for identification, in which case the left wings are removed and vouchered instead.

148 The remainder of the body is returned to its tube, or placed in a new tube if it was originally

149 stored in a Whirl-Pak (Fig. 1D, E).

150

151 4. Specimen storage. Specimen tubes containing tissue, ethanol, and labels (Fig. 1D) are marked 152 on the tube lid with the appropriate accession code. This process is carried out on a block of ice 
153 to keep specimens cold. Fisherbrand ${ }^{\circledR}$ marking pens are used to mark the tubes because their ink

154 is resistant to ethanol "bleeding". Labeled tubes are transferred to a VWR $®$ cardboard freezer

155 box where they are stored upright to prevent leakage, and subsequently returned to the $-80^{\circ} \mathrm{C}$

156 freezer. Microcentrifuge tubes are stored in 5.25" x 5.25" x 3.00" boxes $(13.34 \mathrm{~cm} \mathrm{x} 13.34 \mathrm{~cm} \times$

$1577.62 \mathrm{~cm})$, whereas taller centrifuge tubes are stored in $5.25^{\prime \prime}$ x 5.25" x 4.87 " boxes $(13.34 \mathrm{~cm} \mathrm{x}$

$15813.34 \mathrm{~cm} \times 12.37 \mathrm{~cm})$. Boxes are labeled with the corresponding range of accession codes (e.g.

159 "LEP-12345-12426”) on the upper left corner and side of the lid. Two-inch (5.08 cm) cardboard

160 freezer boxes can be used to store 15 and $50 \mathrm{~mL}$ microcentrifuge tubes laterally, but this

161 approach will risk the possibility of ethanol leakage.

162

163 5. Wing vouchering. The wing voucher step uses two different protocols, depending on the size

164 of the insect. Small specimens with forewing length (FWL) ranging 5-35 mm (Fig. 1) are placed

165 in cardboard coin holders. Large Lepidoptera with FWL $>35 \mathrm{~mm}$ that do not fit in coin holders

166 are stored in double-laminated polypropylene bags (Fig. 2). Both approaches preserve the wings

167 and allow for easy viewing of both the dorsal and ventral surfaces without causing additional

168 damage, and provide the opportunity for wings to be scanned and digitized. Wings can be

169 removed from the holders if direct examination is required. The remainder of the body is kept in

170 a cryogenic freezer $\left(-80^{\circ} \mathrm{C}\right)$ for long-term nucleotide preservation. The wing voucher protocol for

171 small and large wings is presented below.

172 Small specimens: Coin holders - For small specimens, wings are transferred from the

173 sterile petri dish to a clean Kimberly-Clark®, Kimwipe ${ }^{\mathrm{TM}}$ in order to remove excess ethanol.

174 Fine dissecting forceps are used to carefully spread each wing, removing any folds or creases, so

175 that the entirety of each wing lies flat against the Kimwipe and is fully visible after it has dried. 
176 Wings should be handled by gripping the base, or if necessary, the costa; other regions are more

177 fragile and subject to tearing or scale loss. Wings should be dried before transferring them to

178 coin holders (see below), as the clear plastic on coin holders can chemically react and cloud

179 when in direct contact with ethanol. Dried wings are then placed in the clear window of a

180 cardboard coin holder (Fig. 1F-J). We use BCW® 2" x 2" (5.08 cm x $5.08 \mathrm{~cm})$ "coin flips",

181 which can be ordered in three different coin sizes: small (“Quarter”), medium ("Dollar"), and

182 large ("Large Dollar"). In order to reduce wing movement within the coin holder, we recommend

183 using the smallest possible window size that will accommodate a given pair of wings. The coin

184 holder is slowly closed and the edges are neatly stapled to keep the wings secure (Fig. 1H).

185 Copies of the corresponding accession code label and locality label (from Step 2) are glued on

186 the top right and bottom right corners of the coin holder (Fig. 1I, J). If taxonomic information is

187 known, this is marked in the upper left corner and simultaneously entered in the collections

188 database.

189

Large specimens: Lamination - Large Lepidoptera also have their wings dried on a

190 Kimwipe after separation from the rest of the body. Dried wings are placed inside a 4" x 6"

191 (10.16 cm x $15.24 \mathrm{~cm}$ ) Biaxially Oriented Polypropylene (BOPP) bag. The bag is then trimmed

192 by hand to fit the dimensions of the wing (Fig. 2A, B). The trimmed bag with the wings is placed

193 within a plastic $\operatorname{Scotch}{ }^{\circledR}$ 5" x 7" $(12.7 \mathrm{~cm}$ x $17.78 \mathrm{~cm})$ thermal laminating pouch. The BOPP bag

194 creates a protective layer for the wings and prevents the pouch plastic from adhering to their

195 surfaces, enabling wings to be removed from the pouch without damaging them or their scales.

196 Locality and accession code labels are also placed in the pouch, at the lower left and upper right

197 corners of the BOPP bag, respectively (Fig. 2C). The pouch is then carefully inserted into a

198 Scotch laminator (dial set at $5 \mathrm{~mL}$ ) and the lamination process is repeated 2-3 times to ensure a 
199 tight seal (Fig. 2D, E). Each voucher is then neatly trimmed to size, leaving the specimen and

200 labels in a rectangle with rounded corners (Fig. 2F-H).

201

202 6. Voucher storage. Vouchers are organized in numerical order using their unique accession code

203 labels, placed into clear pocket pages (ranging from 4-20 pockets per page, depending on

204 voucher size), and stored in 2" $(5.08 \mathrm{~cm})$ three-ring binders with a slanted binder ring (Fig. 2I-

$205 \mathrm{~K})$. Binders are uniquely numbered and labeled with their corresponding range of accession

206 codes and sampling localities. Binder numbers are entered into the collections database so that

207 each sample in the collection can be queried for the location of its wing voucher.

208

209 Results \& Discussion

210

The importance of molecular voucher specimens in modern evolutionary research cannot

211 be overstated. Natural history collections aim to provide a record of Earth's biodiversity and

212 serve as "windows on evolutionary processes" (Holmes et al. 2016). The preservation of

213 specimens for both morphological and molecular research should be considered a fundamental

214 aspect of the mission of natural history collections.

215 Unfortunately, a standard for molecular vouchering is still lacking to this day for most

216 museums. The procedure presented herein provides an effective long-term preservation method

217 for Lepidoptera tissues while simultaneously creating easily accessible, compact morphological

218 vouchers for long-term storage and identification. Putative informative characters are preserved

219 as best as possible, though some scale loss is inevitable, allowing for vouchered wings to be

220 removed if necessary, if more in-depth investigation is required. Our protocol incorporates one

221 pair of wings; in the event that a voucher is lost, the other pair remains preserved with the rest of 
222 the specimen, thereby allowing tissues to be stored permanently with verifiable morphology. It is

223 important to note that when preserved this way wing color and pattern will be retained, since

224 most wing scales do not rub off in the ethanol or during preparation, allowing specimens to used

225 for both traditional and modern taxonomic and phylogenetic studies (genitalia can be easily

226 dissected from these stored specimens using standard $10 \% \mathrm{KOH}$ ).

227 Our proposed method for storage of molecular samples is not meant to replace traditional

228 Lepidoptera preservation methods (pinning, spreading wings, and drying), nor is our approach

229 meant to replace the classic Lepidoptera pinned collection. Instead, it is meant to complement

230 traditional approaches and create a measure for long-term, secure DNA preservation. We have

231 examined the potential for DNA degradation between dried specimens (as described above) and

232 a tissue taken and placed into $\geq 95 \%$ ethanol, and there is no significant difference, with both

233 approaches providing the quality and quantity required for high-throughput sequencing. We

234 propose that our approach be used, for instance, when multiple specimens of the same species

235 are encountered in the field, allowing for specimens to be preserved using both methods. We also

236 realize that our approach is not applicable to all natural history collections worldwide; there are

237 clear disparities in physical and financial resources available for collection preservation in

238 different countries. Securing biodiversity funding resources to establish an international

239 cooperative repository for genomic quality tissues (e.g. seed banks) would serve as an important

240 step to secure tissues for long-term storage.

241 One limitation to our approach is its applicability to small moths (FWL $<5 \mathrm{~mm}$ ).

242 Although we have had success vouchering some of these moths (e.g., Tortricidae and

243 Gelechioidea), most Microlepidoptera have extremely fragile wings that require delicate care and

244 intricate handling. For very small moths, we suggest the protocol of Lopez-Vaamonde et al. 
245 (2012), which entails pinning the moth while fresh, immediately placing the abdomen and legs in

246 a small tube with ethanol, and storing the tube in a cryogenic freezer. Obviously each natural

247 history collection will have their own way of documenting, labeling, and categorizing specimens.

248 Our details and methodology, outlined above, are not "hard and fast" rules, but rather

249 suggestions for which products and approaches have been successfully applied by the authors.

250 We hope that the procedure presented here will provide a standardized methodology for

251 museum collections that seek to build a Lepidoptera molecular collection. Our vouchering

252 system is flexible and not restricted to any particular database format. It can be used with

253 different management databases such as Specify (http://specifysoftware.org/), Symbiota

254 (http://symbiota.org/docs/), and VoSeq (Pena \& Malm 2012). With the move to digitize natural

255 history collections gathering momentum around the world (Holmes et al. 2016), we feel this

256 method provides Lepidoptera natural history collections the opportunity to easily distribute

257 specimen data and images on a global scale, while accurately linking specimens to tissue

258 samples.

259

260 Acknowledgements

261 The authors thank the dedicated volunteers, students, staff and visiting scholars who have

262 put in countless hours to build the molecular collections at the University of Maryland and the

263 University of Florida. 


\section{Figure Legends}

265 Figure 1. Preparation of small to medium-sized Lepidoptera wing vouchers. (A-J) A - specimen 266 is removed from a Whirl-Pak; B \& C - right wings are cut off at the base and removed; D -

267 specimen is placed into a storage tube with $\geq 95 \%$ ethanol for DNA preservation; E - labels are 268 added to the tube; F \& G - wings are placed in the clear window of a coin holder, spread out 269 with forceps; $\mathrm{H}$ - coin holder is closed and edges are stapled to keep the wings secure; I \& J 270 labels are glued on the top right and bottom right corners of the coin holder.

271

272 Figure 2. Preparation of large Lepidoptera wing vouchers. (A-H) A \& B - following removal of

273 the specimen from ethanol, dried wings are placed inside a BOPP bag, which is then trimmed to 274 wing size; $\mathrm{C}$ - trimmed BOPP bag with wings and corresponding labels is placed within a 275 thermal laminating pouch; D \& E - pouch is laminated 2-3 times to ensure a tight seal; F-H 276 voucher is trimmed to size with scissors, cutting around the specimen and labels in a rectangle 277 with slightly rounded edges; I-K - Stored wing vouchers in binders. I - Coin holder wing 278 vouchers for small moths; $\mathrm{J}$ - laminated wing vouchers for large moths. $\mathrm{K}$ - Wing vouchers 279 stored in binders on shelf. 


\section{Figure 1.}
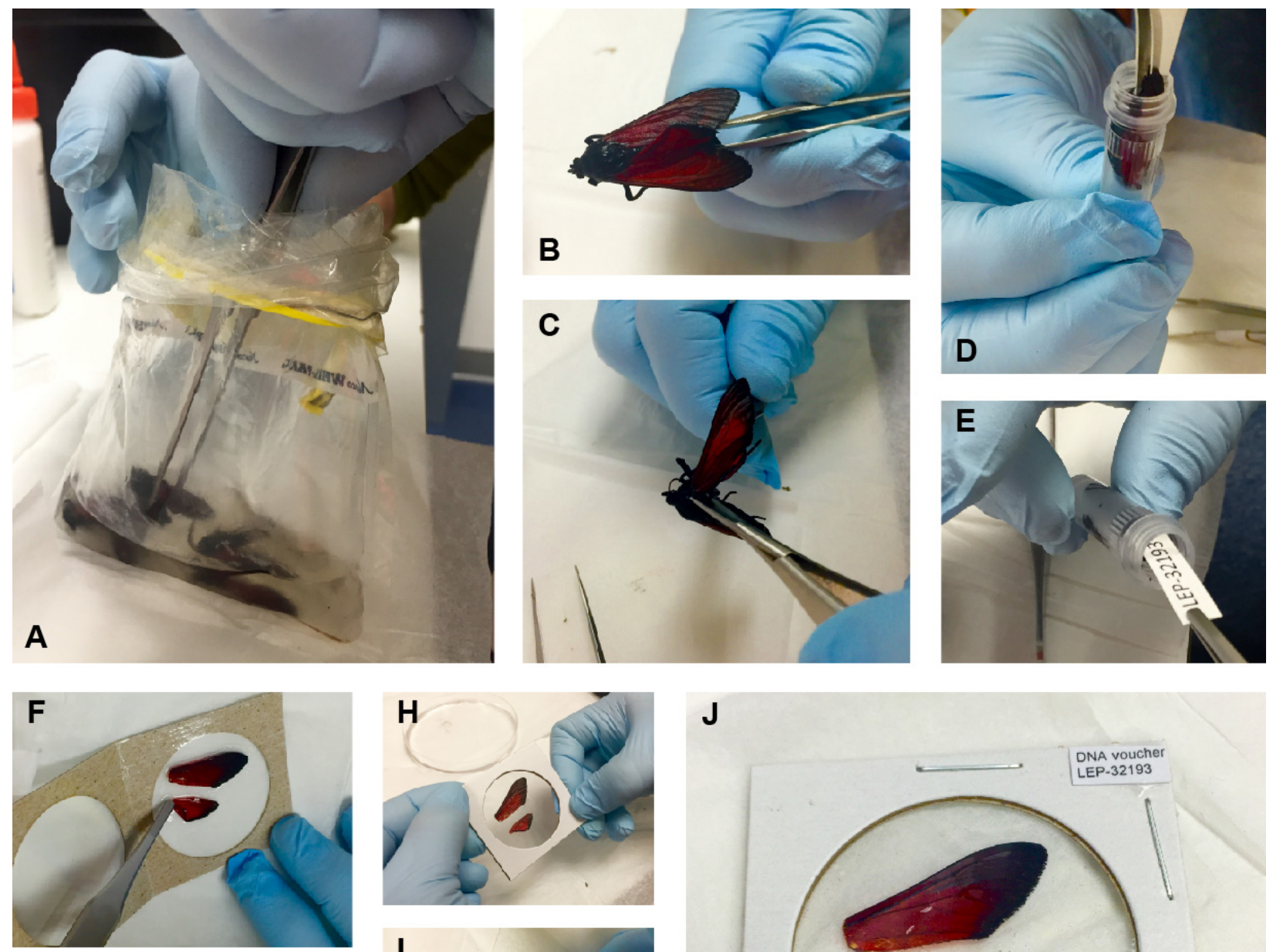

J
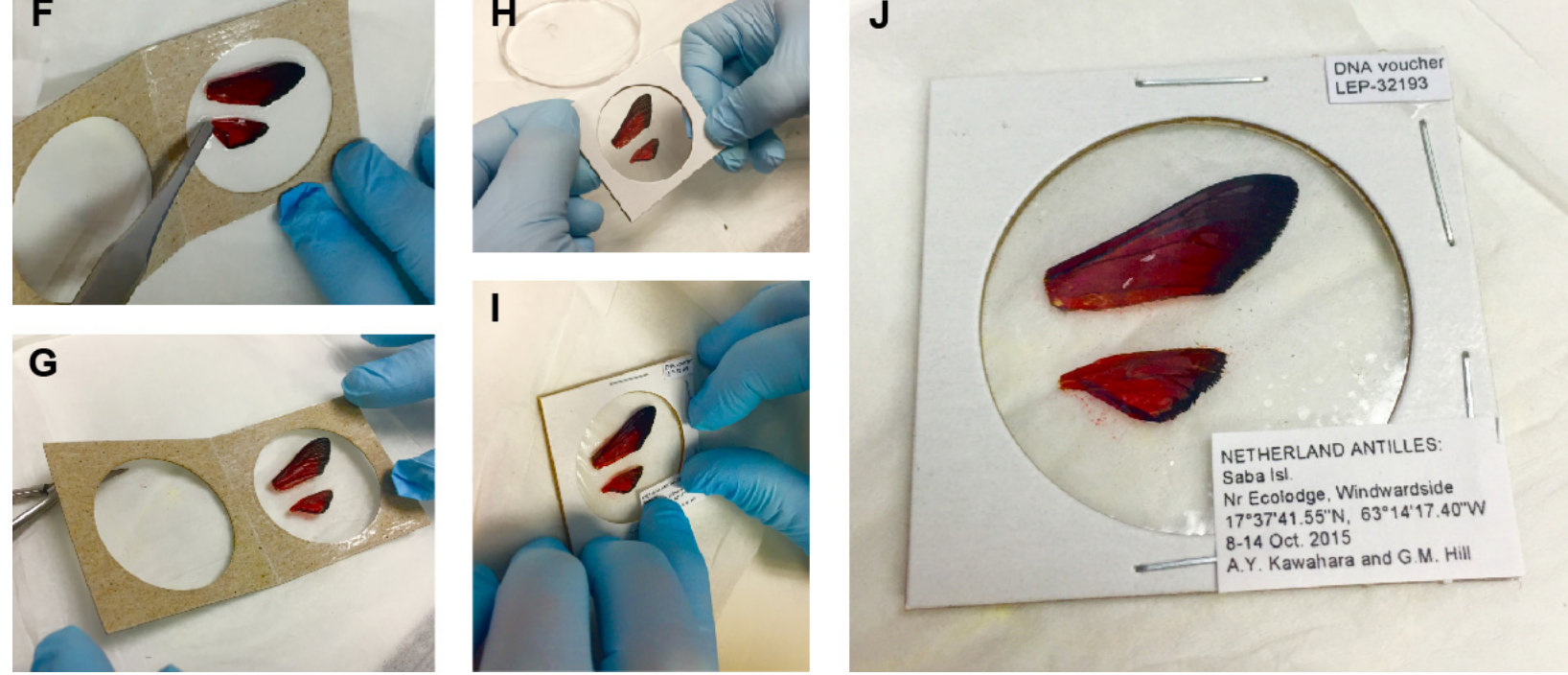


\section{Figure 2.}
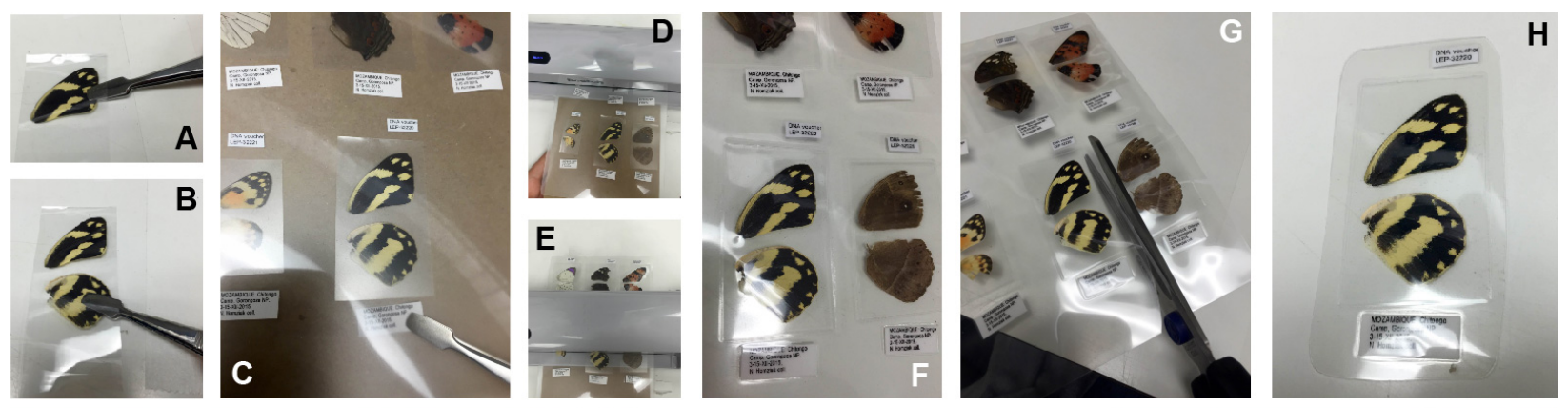

291
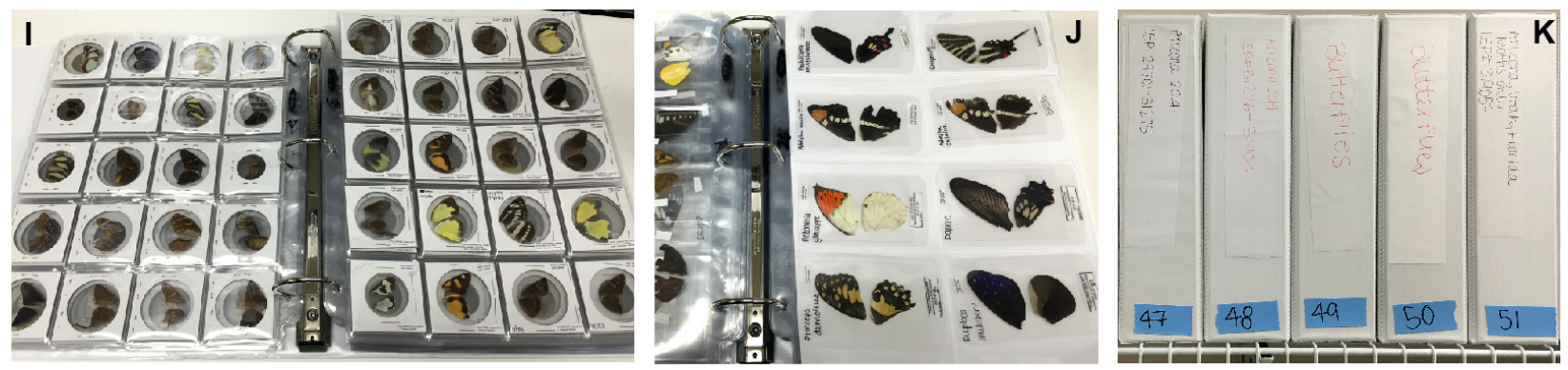

292

293

294

295

296 


\section{References}

298 Bazinet AL, Cummings MP, Mitter KT, and Mitter CW. 2013. Can RNA-Seq resolve the rapid

299

300

301

302

303

304

305

306

307

308

309

310

311

312

313

314

315

316

317

318

319

320

321

322

323

324

325

326

327

328

329

330

331

332

333

334

335

336

337

338

339

340

341

342 radiation of advanced moths and butterflies (Hexapoda: Lepidoptera: Apoditrysia)? An exploratory study. Plos One 8:e82615. UNSP e82615.

Brehm G, and Axmacher JC. 2006. A comparison of manual and automatic moth sampling methods (Lepidoptera : Arctiidae, Geometridae) in a rain forest in Costa Rica. Environmental Entomology 35:757-764.

Brower AVZ. 1994. Phylogeny of Heliconius butterflies inferred from mitochondrial DNA sequences (Lepidoptera: Nymphalidae). Molecular Phylogenetics and Evolution 3:159174.

Brower AVZ. 1996. A new mimetic species of Heliconius (Lepidoptera:Nymphalidae), from southeastern Colombia, revealed by cladistic analysis of mitochondrial DNA sequences. Zoological Journal of the Linnean Society 116:317-332.

Brower AVZ. 2000. Phylogenetic relationships among the Nymphalidae (Lepidoptera) inferred from partial sequences of the wingless gene. Proceedings of the Royal Society of London, Series B, Biological Sciences 267:1201-1211.

Cho S, Zwick A, Regier JC, Mitter C, Cummings MP, Yao J, Du Z, Zhao H, Kawahara AY, Weller S, Davis DR, Baixeras J, Brown JW, and Parr C. 2011. Can deliberately incomplete gene sample augmentation improve a phylogeny estimate for the advanced moths and butterflies (Hexapoda: Lepidoptera)? Syst Biol 60:782-796.

Dean MD, and Ballard JWO. 2001. Factors affecting mitochondrial DNA quality from museum preserved Drosophila simulans. Entomologia Experimentalis Et Applicata 98:279-283.

Dillon N, Austin AD, and Bartowsky E. 1996. Comparison of preservation techniques for DNA extraction from hymenopterous insects. Insect Molecular Biology 5:21-24. DOI 10.1111/j.1365-2583.1996.tb00036.x

Hebert PDN, Penton EH, Burns JM, Janzen DH, and Hallwachs W. 2004. Ten species in one: DNA barcoding reveals cryptic species boundaries in the neotropical skipper butterfly Astraptes fulgerator. Proceedings of the National Academy of Sciences of the United States of America 101:14812-14817. 10.1073/pnas.0406166101

Holmes MW, Hammond TT, Wogan GOU, Walsh RE, LaBarbera K, Wommack EA, Martins FM, Crawford JC, Mack KL, Bloch LM, and Nachman MW. 2016. Natural history collections as windows on evolutionary processes. Molecular Ecology Resources.

Kawahara AY, Emmel TC, Miller J, and Warren AD. 2012. A new institution devoted to insect science: The Florida Museum of Natural History, McGuire Center for Lepidoptera and Biodiversity. Insect Science 19:426-428.

Knolke S, Erlacher S, Hausmann A, Miller MA, and Segerer AH. 2005. A procedure for combined genitalia dissection and DNA extraction in Lepidoptera. Insect Systematics \& Evolution 35:401-409.

Lopez-Vaamonde C, Breman FC, Lees DC, Van Houdt J, and De Prins J. 2012. Analysis of tissue dependent DNA yield for optimal sampling of micro-moths in large-scale biodiversity surveys. European Journal of Entomology 109:1-6.

Pena C, and Malm T. 2012. VoSeq: A Voucher and DNA Sequence Web Application. Plos One 7. 10.1371/journal.pone.0039071

Regier JC, Mitter C, Kristensen NP, Davis DR, Van Nieukerken EJ, Rota J, Simonsen TJ, Mitter KT, Kawahara AY, Yen SH, Cummings MP, and Zwick A. 2015. A molecular phylogeny for the oldest (nonditrysian) lineages of extant Lepidoptera, with implications 
343

344

345

346

347

348

349

350

351

352

353

354

355

356

357

358

359

360

361

362

363

364

365

366

367

368

369

370

371

372

373

for classification, comparative morphology and life-history evolution. Systematic Entomology 40:671-704. 10.1111/syen.12129

Regier JC, Mitter C, Zwick A, Bazinet AL, Cummings MP, Kawahara AY, Sohn JC, Zwickl DJ, Cho S, Davis DR, Baixeras J, Brown J, Parr C, Weller S, Lees DC, and Mitter KT. 2013. A large-scale, higher-level, molecular phylogenetic study of the insect order Lepidoptera (moths and butterflies). Plos One 8:e58568.

Regier JC, Zwick A, Cummings MP, Kawahara AY, Cho S, Weller S, Roe A, Baixeras J, Brown JW, Parr C, Davis DR, Epstein M, Hallwachs W, Hausmann A, Janzen DH, Kitching IJ, Solis MA, Yen SH, Bazinet AL, and Mitter C. 2009. Toward reconstructing the evolution of advanced moths and butterflies (Lepidoptera: Ditrysia): an initial molecular study. Bmc Evolutionary Biology 9:280.

Utrio P. 1983. Sugaring for Moths - Why Are Noctuids Attracted More Than Geometrids. Ecological Entomology 8:437-445.

van Nieukerken EJ, Kaila L, Kitching IJ, Kristensen NP, Lees DC, Minet J, Mitter C, Mutanen M, Regier JC, Simonsen TJ, Wahlberg N, Yen S-H, Zahiri R, Adamski D, Baixeras J, Bartsch D, Bengtsson BÅ, Brown JW, Bucheli SR, Davis DR, De Prins J, De Prins W, Epstein ME, Gentili-Poole P, Gielis C, Hättenschwiler P, Hausmann A, Holloway JD, Kallies A, Karsholt O, Kawahara AY, Koster SJC, Kozlov M, Lafontaine JD, Lamas G, Landry JF, Lee S, Nuss M, Park KT, Penz C, Rota J, Schmidt BC, Schintlmeister A, Sohn JC, Solis MA, Tarmann GM, Warren AD, Weller S, Yakovlev RV, Zolotuhin VV, and Zwick A. 2011. Order Lepidoptera Linnaeus, 1758. In: Zhang, Z.-Q. (Ed.), Animal biodiversity: An outline of higher-level classification and survey of taxonomic richness. Zootaxa 3148:212-221.

Wandeler P, Hoeck PEA, and Keller LF. 2007. Back to the future: museum specimens in population genetics. Trends in Ecology \& Evolution 22:634-642.

Willows-Munro S, and Schoeman MC. 2015. Influence of killing method on Lepidoptera DNA barcode recovery. Molecular Ecology Resources 15:613-618.

Zimmermann M, Wahlberg N, and Descimon H. 2000. Phylogeny of Euphydryas checkerspot butterflies (Lepidoptera : Nymphalidae) based on mitochondrial DNA sequence data. Annals of the Entomological Society of America 93:347-355. 